\title{
Nuclear Receptor Subfamily 1 Group I Member 3
}

National Cancer Institute

\section{Source}

National Cancer Institute. Nuclear Receptor Subfamily 1 Group I Member 3. NCI

Thesaurus. Code C107474.

Nuclear receptor subfamily 1 group I member 3 (352 aa, $\sim 40 \mathrm{kDa}$ ) is encoded by the human NR113 gene. This protein is involved in the regulation of xenobiotic and endobiotic metabolism. 